\title{
MR Product as a Novel Diagnostic Indicator for Chronic Secondary Mitral Regurgitation
}

Mishal Fatima ${ }^{1}$, Nadir Mehmood ${ }^{2}$, Syed Muhammad Jawad Zaidi ${ }^{1}$, Muhammad Hamza ${ }^{1}$, Mehwish Kaneez ${ }^{1}$, Umer Irshad ${ }^{1}$, Muhammad Junaid Azhar ${ }^{1}$, Abdullah Bin Zubair ${ }^{3}$, Rafay Rizwan ${ }^{3}$, Muzammil Sabir $^{3}$

1. Cardiology, Rawalpindi Medical University, Rawalpindi, PAK 2. General Surgery, Rawalpindi Medical University, Rawalpindi, PAK 3. Cardiology, Rashid Latif Medical College, Lahore, PAK

Corresponding author: Syed Muhammad Jawad Zaidi, mjawad927@gmail.com

\begin{abstract}
Background

Chronic secondary mitral regurgitation (SMR) is a common form of valvular heart disease. Its diagnosis through echocardiography is challenging and dependent on subjective interpretations. The subjective error to diagnose SMR can be reduced by developing accurate predictive quantitative parameters that support echocardiographic interpretations and clinical manifestations. The present study aims to develop a new diagnostic indicator for chronic SMR. The new indicator called MR product is the product of left atrial diameter (LAD) and left ventricular internal dimension at end-systole (LVIDs).
\end{abstract}

Materials and Methods

An analytical, case-control study was conducted from transthoracic echocardiography (TTE) reports of 720 patients performed according to the guidelines of the American Society of Echocardiography. The LAD and LVIDs were measured using the standard M Mode TTE. Out of the 720 patients who underwent TTE, 300 patients were diagnosed with chronic SMR by experienced clinicians. Only 115 of those 300 patients met the inclusion criteria for chronic SMR.

Results

The MR product was significantly associated with chronic SMR (rho = 0.83) and predicted it with an odds ratio of 1.014 ( $\mathrm{p}<0.001$ ). The MR product was able to diagnose SMR with a sensitivity of $94.8 \%$ and a specificity of $92.2 \%$, respectively, for a cut off value of $1,045 \mathrm{~mm}^{2}$.

Conclusion

Received 08/12/2020

Review began 08/15/2020 Review ended 08/15/2020 Published 08/26/2020

\section{() Copyright 2020}

Fatima et al. This is an open access article distributed under the terms of the Creative Commons Attribution License CC-BY 4.0., which permits unrestricted use, distribution, and reproduction in any medium, provided the original author and source are credited.
A new parameter called MR product (LAD multiplied with LVIDs) has very high sensitivity and specificity for SMR. Therefore, it can aid in establishing its diagnosis, along with other diagnostic modalities. The new parameter may also potentially increase the diagnostic accuracy of the disease.

Categories: Cardiology, Internal Medicine

Keywords: left atrial diameter (lad), left ventricular internal dimension at end-systole (lvids), secondary mitral regurgitation (smr), mr product

\section{Introduction}

Mitral regurgitation (MR) is a common form of valvular heart disease present in approximately $10 \%$ of adults over 75 years of age [1]. It is categorized based on the disease onset (acute or chronic) and the underlying pathology (primary or secondary). The underlying etiology of chronic secondary mitral regurgitation (SMR) involves functional valve abnormality due to ischemia or heart failure [2]. It is largely asymptomatic in the initial stages, and even in advanced cases (Grades 2 and 3). SMR may present with only mild symptoms, such as exercise intolerance and exertional dyspnea, which do not reflect the severity of the underlying disease process [1-2].

SMR is almost always associated with heart failure (HF) and one in every five patients with HF develops SMR [3]. About $20 \%$ of these patients have severe SMR, which increases the mortality risk by more than two times, while $80 \%$ of patients have a slow, progressive SMR, which is still an independent risk factor for increased mortality in HF patients [3-5]. The higher risk of mortality in such patients is caused by adverse cardiac remodeling due to the development of SMR [4]. Even without heart failure, SMR causes an increased risk of mortality [6]. These findings, combined with the fact the SMR remains largely asymptomatic until late stages, prompts the need for early diagnosis and management of such individuals [2, 7]. A potential area to 
improve these figures is to aid in establishing the diagnosis of chronic SMR with higher confidence so that early interventions can be performed to cure the pathology.

The diagnosis of SMR usually begins with the detection of a heart murmur on routine cardiac examination of a heart failure patient who is typically in Grade 2 or 3 of SMR. Over the years, Doppler flow echocardiography has emerged as the standard method to diagnose a suspected case of SMR [7]. However, the echocardiographic diagnosis is not simple and requires identification of the pattern of associated valvular change, such as asymmetrical or symmetrical tenting, the measurement of coaptation distance (CD) and tenting area (TA), and gauging the degree of backflow of contrast jet, the jet flow area, and vena contracta width by echocardiography reports [8].

The accurate interpretation of a Doppler study requires a sound clinical acumen because it is dependent upon the clinician's subjective assessment. Moreover, interpersonal differences between the interpretations may give rise to false diagnoses [9]. Hence, the dilemma of over or under-reporting of heart pathologies, especially those whose diagnosis is largely based on a physician's subjective opinion on echocardiography reports (such as SMR), is a cause of concern in the medical community and guidelines for the standardization of the technique are regularly revised and published [9-10]. The routine use of Doppler studies further predisposes to subjective errors made by relatively inexperienced clinicians [11]. These diagnostic dilemmas are compounded by the fact that in developing countries, Doppler studies are not readily available in many areas. Therefore, a confirmatory diagnosis cannot be established in such cases [7].

The subjective error in diagnosing certain heart pathologies can be reduced by developing accurate predictive quantitative parameters that support echocardiographic interpretations so that the final diagnosis is not solely based upon the clinician's opinion. One such parameter developed in a study by Haq et al. was the ratio of a left atrial to a left ventricular diastolic diameter which supports the diagnosis of Grade 2 diastolic dysfunction [12]. Thus, it is well-established that the development of quantitative parameters can potentially enhance the echocardiographic diagnosis of cardiac abnormalities.

The present study is concerned with the development of a new diagnostic indicator for chronic SMR. The new indicator is the product of the left atrial diameter (LAD) and the left ventricular internal dimension at end-systole (LVIDs). For the sake of simplicity, the product will be referred to as the MR product in the study. Increased LAD in SMR is well-established [13-14], but its low specificity prevents its use as a diagnostic indicator for heart pathologies. LVIDs are routinely measured in echocardiography and used for the assessment of left ventricular function, along with other ventricular parameters [15]. We could not find any literature that used LVIDs in assessing mitral regurgitation. In our study, the medical reason for using LVIDs in diagnosing secondary mitral regurgitation was the fact that SMR has been invariably associated with left ventricular dysfunction, and therefore, an increase in LVIDs can reflect the development of SMR.

The echocardiography machine measures both diameters of the MR product which minimizes the errors in subjective interpretation. The new indicator, when combined with other modalities, will help in establishing the diagnosis of this condition and may improve the diagnostic accuracy of chronic SMR. It will also help the clinicians to be more confident regarding their opinion on the echocardiography reports of such cases. Thus, the results of this study may add to the diagnostic arsenal for patients with SMR. The MR product can be measured by simple echocardiography (performed without contrast). If it is a valuable diagnostic indicator in our study, the MR product can be used as an initial diagnostic test for SMR, especially in those areas where contrast-based Doppler studies are not readily available.

\section{Materials And Methods}

\section{Study design}

An analytical, case-control study was conducted from March 2017 to September 2018 at the Department of Cardiology of the Holy Family Hospital, Rawalpindi, Pakistan. This study was approved by the Institutional Review Board of Rawalpindi Medical University (IRB-2016-MC-0420).

\section{Echocardiography information}

Transthoracic echocardiography (TTE) reports of 720 patients performed according to the guidelines of the American Society of Echocardiography were collected and documented [10]. The LAD and LVIDs were measured in millimeters $(\mathrm{mm})$ using the standard M Mode TTE and documented. Reports with missing diameters or unclear images were excluded from the study.

\section{Selection of cases}

Three hundred of 720 patients (41.67\%) were diagnosed with chronic SMR by experienced clinicians based on the report of the TTE Doppler echocardiography. We excluded patients with any autoimmune condition, malignancy, structural mitral valve disease (such as stenosis, prolapse, and rheumatic heart disease), mitral repair surgery or mitral valve implant, diastolic dysfunction of any grade, pericarditis, and sepsis from the study. Based on these criteria, 115 cases were then selected. 


\section{Cureus}

\section{Selection of controls}

The patients who underwent Doppler echocardiography but did not have chronic SMR constituted our control population. The same exclusion criteria were applied and 115 controls were then selected after careful age and gender-based matching with the cases.

\section{Statistical techniques}

The descriptive analysis of the study parameters was performed using the IBM Statistical Package for Social Sciences (SPSS), version 23.0 (IBM SPSS Statistics, Armonk, NY). Spearman's correlation was used to observe the correlation between study variables. A Mann-Whitney U test was employed to assess

significant differences in the study variables between cases and controls. A binary logistic regression was performed and the receiver operating characteristic (ROC) curve was constructed to evaluate the predictive power of the MR product for chronic SMR. A p-value of less than 0.05 was considered statistically significant.

\section{Results}

Population parameters and their correlation with heart dimensions, indicated by Spearman's correlation coefficient, are provided in Table 1 . The table shows that the MR product has a stronger correlation (rho = 0.83 ) with chronic SMR than either LAD or LVIDs alone.

\begin{tabular}{|c|c|c|c|c|c|}
\hline \multicolumn{2}{|c|}{ Parameters } & Control group $(n=115)$ & Disease group $(n=115)$ & Spearman correlation rho value (with SMR) & p-value \\
\hline \multirow{2}{*}{ Gender } & Males & 54 & 55 & \multirow{2}{*}{ - } & \multirow{2}{*}{ - } \\
\hline & Females & 61 & 60 & & \\
\hline \multicolumn{2}{|l|}{ Age } & $51.26 \pm 18.13$ & $54.13 \pm 16.34$ & - & - \\
\hline \multicolumn{2}{|l|}{ LAD (mm) } & $30.79 \pm 4.41$ & $39.55 \pm 5.8$ & 0.694 & $<0.001$ \\
\hline \multicolumn{2}{|c|}{ LVIDs (mm) } & $27.63 \pm 4.16$ & $37.98 \pm 6.20$ & 0.71 & $<0.001$ \\
\hline \multicolumn{2}{|c|}{ MR Product (LADLVIDs) } & $850.56 \pm 171.49$ & $1503.61 \pm 338.78$ & 0.833 & $<0.001$ \\
\hline
\end{tabular}

\section{TABLE 1: Population Parameters and Their Correlation With Chronic SMR}

LAD: left atrial diameter; LVIDs: left ventricular internal dimension at end-systole; SMR: secondary mitral regurgitation

Table 2 delineates the result of the Mann-Whitney $\mathrm{U}$ test. The mean rank was greater in SMR patients for LAD, LVIDs, and the MR product. This was highly significant $(\mathrm{p}<0.001)$, indicating that larger values of the three parameters are significantly associated with SMR.

\begin{tabular}{|l|lll|}
\hline Parameter & Mean Rank & & \\
\hline & NO SMR & SMR & \\
\hline LAD & 69.30 & 161.30 & $<0.001$ \\
\hline LVIDs & 66.30 & 164.70 & $<0.001$ \\
\hline MR Product (LAD*LVIDs) & 60.22 & 170.78 & $<0.001$ \\
\hline
\end{tabular}

\section{TABLE 2: Results of the Mann-Whitney U Test for Three Parameters}

LAD: left atrial diameter; LVIDs: left ventricular internal dimension at end-systole; SMR: secondary mitral regurgitation

Table 3 elucidates the results of binary logistic regression. The odds ratio was 1.014 which was highly significant $(\mathrm{p}<0.001)$. 


\section{Cureus}

\section{Variable}

MR product (LAD*LVIDs)
Regression Coefficient (B)

0.014
Odds Ratio (Exp B)

1.014
$95 \% \mathrm{CI}$

$1.010-1.080$ p-value

$<0.001$

\section{TABLE 3: Binary Logistic Regression Model for SMR}

$\mathrm{Cl}$ : confidence interval; Exp B: exponentiation of the B coefficient (odds ratio); LAD: left atrial diameter; LVIDs: left ventricular internal dimension at end-systole

Table 4 shows that our binary logistic model correctly predicts $90.7 \%$ of cases as compared to $50 \%$ of cases of the null model.

\begin{tabular}{|c|c|c|c|c|}
\hline \multirow{3}{*}{\multicolumn{2}{|c|}{ Observed }} & \multicolumn{3}{|l|}{ Predicted } \\
\hline & & \multicolumn{2}{|l|}{ Diagnosis } & \multirow{2}{*}{ Percentage Correct } \\
\hline & & No SMR & SMR & \\
\hline \multirow{2}{*}{ Diagnosis } & No SMR & 109 & 6 & 94.8 \\
\hline & SMR & 15 & 100 & 87.0 \\
\hline \multicolumn{4}{|c|}{ Overall percentage } & 90.9 \\
\hline \multicolumn{5}{|c|}{ The value of Nagelkerke $R^{2}$ was 0.84} \\
\hline \multicolumn{5}{|c|}{ The predictive capacity of $t$} \\
\hline
\end{tabular}

\section{TABLE 4: Predictive Capability of Binary Logistic Regression}

SMR: secondary mitral regurgitation

The receiver operating characteristic (ROC) curve characteristics of the three parameters (LAD, LVIDs, and MR product) are provided in Table 5 . The area under the curve was highest for MR product at 0.981 , showing a very high predictive power of the MR product for chronic SMR.

\begin{tabular}{|c|c|c|c|c|c|c|}
\hline Parameter & Area & $\begin{array}{l}\text { p- } \\
\text { value }\end{array}$ & $95 \% \mathrm{Cl}$ & $\begin{array}{l}\text { Selected cut-off } \\
\text { value }\end{array}$ & $\begin{array}{l}\text { Sensitivity at the cut-off } \\
\text { value }\end{array}$ & Specificity at the cut-off value \\
\hline LAD & 0.689 & 0.004 & $\begin{array}{l}0.65- \\
0.71\end{array}$ & $43.5 \mathrm{~mm}$ & $75.7 \%$ & $61.8 \%$ \\
\hline LVIDs & 0.754 & $<0.001$ & $\begin{array}{l}0.72- \\
0.78\end{array}$ & $38.5 \mathrm{~mm}$ & $71.4 \%$ & $69.5 \%$ \\
\hline $\begin{array}{l}\text { MR } \\
\text { product (LAD*LVIDs) }\end{array}$ & 0.981 & $<0.001$ & $\begin{array}{l}0.97- \\
0.99\end{array}$ & $1,045.00 \mathrm{~mm}^{2}$ & $94.8 \%$ & $92.2 \%$ \\
\hline
\end{tabular}

\section{TABLE 5: Receiver Operating Characteristic (ROC) Curve of the Three Parameters}

Cl: confidence interval; LAD: left atrial diameter; LVIDs: left ventricular internal dimension at end-systole

\section{Discussion}

Despite the heavy clinical burden of chronic secondary mitral regurgitation, its diagnosis and management offer many challenges, and long-term outcomes of surgical procedures have not been well-established, even in developed countries [16]. In developing countries, where contrast-based studies are readily accessible only at tertiary health care centers, the diagnosis rate of chronic SMR is even lower and many patients are diagnosed in the advanced irreversible stage associated with heart failure [7]. Although therapeutic interventions change the disease course of mitral regurgitation if instituted early, only a very few 
patients receive timely treatment. A study reported that only about half of the patients with primary MR received adequate therapeutic intervention [17]. While no statistics were found regarding the treatment ratio in SMR, it is presumed to be low as well. The lack of health education and moderate intensity of the symptoms add to the dismal picture surrounding this important cardiac pathology. Our study is an effort to aid in the diagnosis of chronic SMR, especially in areas where Doppler studies are not easily accessible, so that timely therapeutic interventions can be performed and better health care can be provided to these patients.

The MR product employs two heart dimensions, LAD and LVIDs. Many studies have correlated LAD with chronic SMR and a few studies have established left atrial enlargement as a contributing factor for SMR [14]. Whether it's a contributing factor for SMR or only an associated finding due to underlying left ventricular dysfunction is still yet to be determined. Enlargement of the left atrium is a routine finding on the echocardiography of SMR patients $[14,18]$. Our results agree with these findings and the logical medical explanation is compensatory left atrial hypertrophy and dilatation in response to high-pressure backflow through the incompetent mitral valve.

When used alone as a diagnostic marker of SMR, the ROC curve of LAD shows sensitivity and specificity of $75.7 \%$ and $61.8 \%$, respectively, at a cut off value of $43.5 \mathrm{~mm}$, whereas the normal accepted left atrial diameter is $33.6 \mathrm{~mm} \pm 4.1$ [19]. The reason behind the low specificity and sensitivity is multifactorial causation of increased LAD, such as diastolic dysfunction, the normal aging process, hypertension, and obesity, which decrease its predictive accuracy for chronic SMR [20-21]. Therefore, although LAD is an independent risk factor for survival in SMR patients [22], its diagnostic utility is limited because of relatively low specificity. However, because of its clinical importance, LAD combined with other heart dimensions has been used as a marker for various heart pathologies [12]. It was employed as a ratio with left ventricular volume in a Chinese study for predicting hypertension-specific atrial enlargement [23]. Its role in predicting diastolic dysfunction was established in another study at Rawalpindi Institute of Cardiology [12]. Nevertheless, its role in the assessment of mitral regurgitation has not been studied. In our study, LVIDs shows a sensitivity and specificity of $71.4 \%$ and $69.5 \%$, respectively, for chronic SMR, at a cut off value of $38.5 \mathrm{~mm}$, whereas the normal accepted LVIDs is $29.9 \pm 4.7$ [19].

The new parameter uses the product of these two dimensions and shows a marked increase in predictive accuracy and strength of correlation than either LAD or LVIDs alone. The rho value of 0.83 indicates a very high strength of correlation with chronic SMR. At the area under the curve (AOC) of 0.98, the optimal sensitivity and specificity are $92.2 \%$ and $94.8 \%$, respectively, for a cut off value of $1,045 \mathrm{~mm}^{2}$. This high predictive power is further validated by a binary logistic model which reports a predictive accuracy of $90.1 \%$ and Nagelkerke $\mathrm{R}^{2}$ of 0.84 , meaning that our model, based on the MR product as a diagnostic indicator, is accurate in $90 \%$ of cases and explains $84 \%$ of the variability in the observations. The odds ratio of 1.014 was also highly significant $(\mathrm{p}<0.001)$, indicating a strong relationship between the MR product and SMR. Thus, the statistics provide a strong logical basis for using the MR product as a diagnostic indicator of chronic SMR.

Based on these statistics, the MR product has the potential for many clinical applications in SMR. It may improve diagnostic accuracy when combined with other modalities, such as echocardiography and electrocardiography. It can be also be used for screening of suspected individuals before ordering a detailed contrast-based study, especially in areas where Doppler studies are expensive and not readily available. This application can increase the diagnostic rate and reduce health care burden by earlier diagnosis, timely interventions, and eventually, reduced mortality rates in such areas.

The study has certain limitations. Firstly, the diagnostic accuracy of the MR product needs to be confirmed by large clinical trials involving many geographic areas and it should be correlated with different patient parameters. Secondly, although the MR product may increase the diagnostic accuracy, its routine clinical use will require the proof of improved diagnostic rate of SMR by multiple studies.

\section{Conclusions}

Chronic secondary mitral regurgitation (SMR) is a relatively prevalent condition with numerous diagnostic challenges and a poor clinical outcome when left undiagnosed and untreated. A new parameter called the MR product (LAD multiplied with LVIDs) has a very high sensitivity and specificity of $92.2 \%$ and $94.8 \%$, respectively, for this condition. It can aid in establishing the diagnosis of SMR, along with other modalities, and may potentially increase the diagnostic accuracy for the disease. The MR product can also be used as a screening test for SMR in areas where contrast-based studies are not accessible on a large scale, thus ensuring better medical care for patients in these areas.

\section{Additional Information \\ Disclosures}

Human subjects: Consent was obtained by all participants in this study. Institutional Review Board of Rawalpindi Medical University issued approval IRB-2016-MC-042. Animal subjects: All authors have 
confirmed that this study did not involve animal subjects or tissue. Conflicts of interest: In compliance with the ICMJE uniform disclosure form, all authors declare the following: Payment/services info: All authors have declared that no financial support was received from any organization for the submitted work. Financial relationships: All authors have declared that they have no financial relationships at present or within the previous three years with any organizations that might have an interest in the submitted work.

Other relationships: All authors have declared that there are no other relationships or activities that could appear to have influenced the submitted work.

\section{References}

1. Maganti K, Rigolin VH, Sarano ME, Bonow RO: Valvular heart disease: diagnosis and management. Mayo Clin Proc. 2010, 85:483-500. 10.4065/mcp.2009.0706

2. Coleman W, Weidman-Evans E, Clawson R: Diagnosing and managing mitral regurgitation. JAAPA. 2017, 30:11-14. 10.1097/01.JAA.0000516342.41351.6d

3. Bartko PE, Pavo N, Pérez-Serradilla A, et al.: Evolution of secondary mitral regurgitation. Eur Heart J Cardiovasc Imaging. 2018, 19:622-629. 10.1093/ehjci/jey023

4. Asgar AW, Mack MJ, Stone GW: Secondary mitral regurgitation in heart failure: pathophysiology, prognosis, and therapeutic considerations. J Am Coll Cardiol. 2015, 65:1231-1248. 10.1016/j.jacc.2015.02.009

5. Obadia JF, Messika-Zeitoun D, Leurent G, et al.: Percutaneous repair or medical treatment for secondary mitral regurgitation. N Engl J Med. 2018, 379:2297-2306. 10.1056/NEJMoa1805374

6. Sannino A, Smith RL 2nd, Schiattarella GG, Trimarco B, Esposito G, Grayburn PA: Survival and cardiovascular outcomes of patients with secondary mitral regurgitation: a systematic review and metaanalysis. JAMA Cardiol. 2017, 2:1130-1139. 10.1001/jamacardio.2017.2976

7. Cardiovascular Imaging in the Developing World . (2016). Accessed: August 23, 2020: http://www.cathlabdigest.com/article/Cardiovascular-Imaging-Developing-World.

8. Lancellotti P, Zamorano JL, Vannan MA: Imaging challenges in secondary mitral regurgitation: unsolved issues and perspectives. Circ Cardiovasc Imaging. 2014, 7:735-746. 10.1161/CIRCIMAGING.114.000992

9. Evangelista A, Flachskampf F, Lancellotti P, et al.: European Association of Echocardiography recommendations for standardization of performance, digital storage and reporting of echocardiographic studies. Eur J Echocardiogr. 2008, 9:438-448. 10.1093/ejechocard//ien174

10. Mitchell C, Rahko PS, Blauwet LA, et al.: Guidelines for performing a comprehensive transthoracic echocardiographic examination in adults: recommendations from the American Society of Echocardiography. J Am Soc Echocardiogr. 2019, 32:1-64. 10.1016/j.echo.2018.06.004

11. Benavidez OJ, Gauvreau K, Geva T: Diagnostic errors in congenital echocardiography: importance of study conditions. J Am Soc Echocardiogr. 2014, 27:616-623. 10.1016/j.echo.2014.03.001

12. Haq IU, Saeed M, Hamza M, Ali AS, Nadir M: Left atrial to left ventricular end diastolic diameter ratio in left ventricular diastolic dysfunction. Pak J Physiol. 2017, 13:15-18.

13. Abhayaratna WP, Seward JB, Appleton CP, Douglas PS, Oh JK, Tajik AJ, Tsang TSM: Left atrial size: physiologic determinants and clinical applications. J Am Coll Cardiol. 2006, 47:2357-2363. 10.1016/j.jacc.2006.02.048

14. Park SM, Park SW, Casaclang-Verzosa G, et al.: Diastolic dysfunction and left atrial enlargement as contributing factors to functional mitral regurgitation in dilated cardiomyopathy: data from the Acorn trial. Am Heart I. 2009, 157:762.e3-762.e10. 10.1016/i.ahi.2008.12.018

15. Chengode S: Left ventricular global systolic function assessment by echocardiography . Ann Card Anaesth. 2016, 19:S26-S34. 10.4103/0971-9784.192617

16. Nishimura RA, Vahanian A, Eleid MF, Mack MJ: Mitral valve disease-current management and future challenges. Lancet. 2016, 387:1324-1334. 10.1016/S0140-6736(16)00558-4

17. Wu S, Chai A, Arimie S, Mehra A, Clavijo L, Matthews RV, Shavelle DM: Incidence and treatment of severe primary mitral regurgitation in contemporary clinical practice. Cardiovasc Revasc Med. 2018, 19:960-963. 10.1016/i.carrev.2018.07.021

18. Patel DA, Lavie CJ, Milani RV, Shah S, Gilliland Y: Clinical implications of left atrial enlargement: a review . Ochsner J. 2009, 9:191-196.

19. Kou S, Caballero L, Dulgheru R, et al.: Echocardiographic reference ranges for normal cardiac chamber size: results from the NORRE study. Eur Heart J Cardiovasc Imaging. 2014, 15:680-690. 10.1093/ehjci/jet284

20. Ou Q, Chen Y, Yu S, Guo X, Zhao H, Sun Y: Prevalence of left atrial enlargement and its risk factors in general Chinese population. BMC Cardiovasc Disord. 2016, 16:53. 10.1186/s12872-016-0229-z

21. Bombelli M, Cuspidi C, Facchetti R, et al.: New-onset left atrial enlargement in a general population . I Hypertens. 2016, 34:1838-1845. 10.1097/HJH.0000000000001022

22. Rusinaru D, Tribouilloy C, Grigioni F, et al.: Left atrial size is a potent predictor of mortality in mitral regurgitation due to flail leaflets: results from a large international multicenter study. Circ Cardiovasc Imaging. 2011, 4:473-481. 10.1161/circimaging.110.961011

23. Li Y, Chai L, Zhang Y, Li L: Novel parameter for assessment of left atrial size in patients with hypertension: ratio of left atrial volume to left ventricular volume. Chin Med J (Engl). 2009, 122:2325-2329.

10.3760/cma.j.issn.0366-6999.2009.19.025 